TITLE:

\title{
Analyzing atomic liquids and solids by means of two-dimensional Raman spectra in frequency domain
}

\author{
$\operatorname{AUTHOR}(\mathrm{S})$ :
}

Nagata, Y; Hasegawa, T; Tanimura, Y

\section{CITATION:}

Nagata, Y ... [et al]. Analyzing atomic liquids and solids by means of two-dimensional Raman spectra in frequency domain. JOURNAL OF CHEMICAL PHYSICS 2006, 124(19): 194504.

ISSUE DATE:

2006-05-21

URL:

http://hdl.handle.net/2433/49828

\section{RIGHT:}

Copyright 2006 American Institute of Physics. This article may be downloaded for personal use only. Any other use requires prior permission of the author and the American Institute of Physics. 


\title{
Analyzing atomic liquids and solids by means of two-dimensional Raman spectra in frequency domain
}

\author{
Yuki Nagata, ${ }^{\text {a) }}$ Taisuke Hasegawa, and Yoshitaka Tanimura \\ Department of Chemistry, Graduate School of Science, Kyoto University, Sakyoku, Kyoto 606-8502, Japan
}

(Received 11 January 2006; accepted 10 March 2006; published online 17 May 2006)

\begin{abstract}
A practical method to evaluate the contributions of the nonlinear polarizability and anharmonicity of potentials from the experimental and simulation data by using double Fourier transformation is presented. In a Lennard-Jones potential system, an approximated expression of the fifth-order response function using the ratio between nonlinear polarizability and anharmonicity exhibits a good agreement with the results of the molecular dynamics simulation. In a soft-core case, the fifth-order Raman signal indicates that the system consists of the delocalized and localized modes, and only the delocalized mode affects the dramatic change of the fifth-order Raman response functions between solid and liquid phases through nonlinear polarizability. () 2006 American Institute of Physics. [DOI: 10.1063/1.2191850]
\end{abstract}

\section{INTRODUCTION}

Nonlinear optical interactions between the laser and molecular system provide valuable and versatile spectroscopic information to understand the dynamics of the system as well as its environment. For molecules in a condensed phase, fifth-order Raman and third-order infrared (IR) spectroscopies allow us to capture greater detail in molecular dynamics and structure than third-order Raman and firstorder IR spectroscopies. ${ }^{1}$ The utility and possibility of these spectroscopies have been demonstrated by various approaches including theoretical analyse ${ }^{2-7}$ molecular dynamics (MD) simulations, ${ }^{8-21}$ ab initio calculations, ${ }^{22,23}$ and a variety of experiments. ${ }^{24-33}$ Although the potential of multidimensional vibrational spectroscopies is now well recognized, our comprehension of two-dimensional (2D) contour maps has not been achieved completely. In a fifth-order Raman case, this might be attributed to the lack of theory explaining an overall profile of signal. Our previous paper ${ }^{11}$ showed how one could utilize the symmetric and antisymmetric integrated response functions ${ }^{34}$ to characterize the role of stability matrix in one-dimensional (1D) plots. These functions cannot separate the contribution of nonlinear polarizability from that of anharmonicity of potentials but isolate the contribution of the stability matrix from that of the simple three-body correlation function. The role of stability matrix has an important meaning in terms of equilibrium simulation, whereas the comparison of the term of nonlinear polarizability with that of anharmonicity is intriguing with respect to the normal mode (NM) analysis. ${ }^{8,10,14,15}$

This paper presents a practical method to evaluate the relative contributions of nonlinear polarizability and anharmonicity of potentials from the experimental and simulation data. Although these contributions can be estimated from analytical theories ${ }^{3}$ or MD simulations ${ }^{10,15}$ by turning on and off the terms responsible for these contributions, it has not

${ }^{a)}$ Electronic mail: nagata@kuchem.kyoto-u.ac.jp been possible to analyze directly the experimental data especially for multimode systems due to the complication of the 2D maps. Our approach can extract quantitative information about the ratio between the nonlinear polarizability and anharmonicity of potentials in the molecular system. To evaluate this ratio, we have derived the analytical expression of the fifth-order Raman signal for an anharmonic potential system based on a perturbative calculation of a Morse oscillator system. ${ }^{3,15}$ By carrying out the double Fourier transformation of the 2D time domain Raman signal, we have obtained the frequency domain expressions of the fifth-order Raman signal. The analysis of spectral volumes gives access to the ratio between nonlinear polarizability and anharmonicity, which reveals the change of molecular mechanisms between solid and liquid phases. ${ }^{11}$

In Sec. II, we have derived the expression of fifth-order response functions in the frequency domain. The derivation of the analytical expression for anharmonicity term is explained in Appendix. In Sec. III, we have applied our method to simple liquids described by a Lennard-Jones (LJ) potential and showed that we can clarify each vibrational mode uniquely by using $2 \mathrm{D}$ frequency domain maps. The NM expressions using the calculated ratio between nonlinear polarizability and anharmonicity are compared with MD simulation results. In Sec. IV, we further apply our method to the soft-core potential systems to investigate a role of nonlinear polarizability and anharmonicity, which was not clarified in the previous study. ${ }^{11}$ Section V is devoted to the concluding remarks.

\section{FREQUENCY DOMAIN SIGNALS OF FIFTH-ORDER RAMAN PROCESS}

The fifth-order Raman response function is defined by a three-body correlation function of polarizability. In this section, we demonstrate how one can obtain the information of 
the nonlinear polarizability and anharmonicity from the experimental and simulation data by using the analytical expression of the response function.

We assume that the polarizability with $a b$ tensor $\Pi_{a b}(t)$ is expanded in terms of a single molecular coordinate $q(t)$ as

$$
\Pi_{a b}(t)-\Pi_{a b}=\Pi_{a b}^{\prime} q(t)+\frac{\lambda}{2} \Pi_{a b}^{\prime \prime} q(t)^{2}+\cdots,
$$

where $\lambda$ is a perturbation index which is set to unity after completion of the perturbation expansion. The molecular coordinate is treated as a harmonic motion $q_{H}(t)$ plus a perturbative anharmonic motion $q_{A}(t)$,

$$
q(t)=q_{H}(t)+\lambda q_{A}(t)
$$

The fifth-order response function $R_{a b c d e f}^{(5)}\left(t_{2}, t_{1}\right)$ is expressed as

$$
\begin{aligned}
R_{a b c d e f}^{(5)}\left(t_{2}, t_{1}\right)= & R_{a b c d e f, \mathrm{LT}}^{(5)}\left(t_{2}, t_{1}\right) \\
& +\lambda\left(R_{a b c d e f, \mathrm{NL}}^{(5)}\left(t_{2}, t_{1}\right)+R_{a b c d e f, \mathrm{AN}}^{(5)}\left(t_{2}, t_{1}\right)\right),
\end{aligned}
$$

where

$$
R_{a b c d e f, \mathrm{LT}}^{(5)}\left(t_{2}, t_{1}\right)=\frac{1}{k T}\left\langle\Pi_{a b}^{\prime} \Pi_{c d}^{\prime} \Pi_{e f}^{\prime} M_{H}\left(t_{2}, 0\right) \dot{q}_{H}\left(-t_{1}\right)\right\rangle
$$

and

$$
\begin{aligned}
R_{a b c d e f, \mathrm{NL}}^{(5)}\left(t_{2}, t_{1}\right)= & \frac{1}{k T}\left\langle\Pi_{a b}^{\prime \prime} \Pi_{c d}^{\prime} \Pi_{e f}^{\prime} M_{H}\left(t_{2}, 0\right) q_{H}\left(t_{2}\right) \dot{q}_{H}\left(-t_{1}\right)\right\rangle \\
& +\frac{1}{k T}\left\langle\Pi_{a b}^{\prime} \Pi_{c d}^{\prime \prime} \Pi_{e f}^{\prime} M_{H}\left(t_{2}, 0\right) q_{H}(0) \dot{q}_{H}\left(-t_{1}\right)\right\rangle \\
& +\frac{1}{k T}\left\langle\Pi_{a b}^{\prime} \Pi_{c d}^{\prime} \Pi_{e f}^{\prime \prime} M_{H}\left(t_{2}, 0\right) q_{H}\left(-t_{1}\right) \dot{q}_{H}\left(-t_{1}\right)\right\rangle
\end{aligned}
$$

are the contributions from linear and nonlinear polarizabilities and

$$
\begin{aligned}
R_{a b c d e f, \mathrm{AN}}^{(5)}\left(t_{2}, t_{1}\right)= & \frac{1}{k T}\left\langle\Pi_{a b}^{\prime} \Pi_{c d}^{\prime} \Pi_{e f}^{\prime} M_{A}\left(t_{2}, 0\right) \dot{q}_{H}\left(-t_{1}\right)\right\rangle \\
& +\frac{1}{k T}\left\langle\Pi_{a b}^{\prime} \Pi_{c d}^{\prime} \Pi_{e f}^{\prime} M_{H}\left(t_{2}, 0\right) \dot{q}_{A}\left(-t_{1}\right)\right\rangle
\end{aligned}
$$

is that from anharmonicity of potentials. Here, $M\left(t_{2}, t_{1}\right)$ $=M_{H}\left(t_{2}, t_{1}\right)+\lambda M_{A}\left(t_{2}, t_{1}\right)$ is the stability matrix for harmonic and anharmonic parts of trajectories, and $k T$ represents the temperature multiplied by the Boltzmann constant.

In the Brownian oscillator (BO) model, the linear polarizability term vanishes and the nonlinear polarizability term is reduced to ${ }^{2,3,8}$

$$
\begin{aligned}
& R_{a b c d e f, \mathrm{NL}}^{(5)}\left(t_{2}, t_{1}\right) \\
& \propto\left\langle\left\langle\Pi_{a b}^{\prime} \Pi_{c d}^{\prime \prime} \Pi_{e f}^{\prime}\right\rangle \int d \omega \frac{\rho(\omega)}{\omega^{2}} C^{\prime \prime}\left(t_{2}\right) C^{\prime \prime}\left(t_{1}\right)\right. \\
& \quad+\left\langle\Pi_{a b}^{\prime \prime} \Pi_{c d}^{\prime} \Pi_{e f}^{\prime}\right\rangle \int d \omega \frac{\rho(\omega)}{\omega^{2}} C^{\prime \prime}\left(t_{2}\right) C^{\prime \prime}\left(t_{1}+t_{2}\right),
\end{aligned}
$$

where $C^{\prime \prime}(t)=\sin (\omega t) e^{-\gamma}$ and the decay rate $\gamma$ is assumed to be independent of $\omega$ in order to simplify the following discussion. Hereafter, we adopt the BO model with an exponential decay and do not take a biexponential decay. The expressions of decay functions do not make much difference on the following procedure and discussion about the ratio between nonlinear polarizability and anharmonicity of potentials.

Since the fifth-order signal calculated from the BO approach does not involve the anharmonic contribution, it disagrees with the direct evaluation of the response function by means of MD simulations. In order to improve the BO approach and evaluate $R_{a b c d e f, \mathrm{AN}}^{(5)}\left(t_{2}, t_{1}\right)$, we analytically calculate $q_{H}(t)$ and $q_{A}(t)$ for a Morse potential

$$
V(q)=D\left(e^{-2 \beta q}-2 e^{-\beta q}\right)
$$

from the perturbative expansion approach, where $\beta$ and $D$ are potential parameters. We then derived the anharmonicity part of the fifth-order response function as

$$
R_{a b c d e f, \mathrm{AN}}^{(5)}\left(t_{2}, t_{1}\right)
$$

$$
\propto \beta\left\langle\Pi_{a b}^{\prime} \Pi_{c d}^{\prime} \Pi_{e f}^{\prime}\right\rangle \int d \omega \frac{\rho(\omega)}{\omega^{2}} 4 C^{\prime \prime 3}\left(\frac{t_{2}}{2}\right) C^{\prime \prime}\left(t_{1}+\frac{t_{2}}{2}\right) .
$$

An explicit derivation of Eq. (9) is given in Appendix, where we compare Eq. (9) with the anharmonic part of the fifthorder response function for the anharmonicity additive potential and show the accordance of Eq. (9) with the expressions obtained by Okumura and Tanimura ${ }^{3}$ and by Ma and Stratt ${ }^{15}$ for qubic anharmonicity $g_{3}=-6 D \beta^{3}$

The anharmonic term (9), together with the nonlinear term (7), casts the total fifth-order response function into the form

$$
\begin{aligned}
& R_{\text {abcdef }}^{(5)}\left(t_{2}, t_{1}\right) \\
& \propto\left\langle\Pi_{a b}^{\prime} \Pi_{c d}^{\prime \prime} \Pi_{e f}^{\prime}\right\rangle \int d \omega \frac{\rho(\omega)}{\omega^{2}} C^{\prime \prime}\left(t_{2}\right) C^{\prime \prime}\left(t_{1}\right) \\
& \quad+\left\langle\Pi_{a b}^{\prime \prime} \Pi_{c d}^{\prime} \Pi_{e f}^{\prime}\right\rangle \int d \omega \frac{\rho(\omega)}{\omega^{2}} C^{\prime \prime}\left(t_{2}\right) C^{\prime \prime}\left(t_{1}+t_{2}\right) \\
& \quad-\beta\left\langle\Pi_{a b}^{\prime} \Pi_{c d}^{\prime} \Pi_{e f}^{\prime}\right\rangle \int d \omega \frac{\rho(\omega)}{\omega^{2}} 4 C^{\prime \prime 3}\left(\frac{t_{2}}{2}\right) C^{\prime \prime}\left(t_{1}+\frac{t_{2}}{2}\right) .
\end{aligned}
$$

If $a=c$ and $b=d$, we can recast Eq. (10) as 


$$
\begin{aligned}
R_{a b c d e f}^{(5)}\left(t_{2}, t_{1}\right) \propto & \int d \omega \frac{\rho(\omega)}{\omega^{2}} \\
& \times\left\{k_{\text {abcdef, } \mathrm{NL}}(\omega) C^{\prime \prime}\left(t_{2}\right)\left(C^{\prime \prime}\left(t_{1}\right)+C^{\prime \prime}\left(t_{1}+t_{2}\right)\right)\right. \\
& \left.+k_{a b c d e f, \mathrm{AN}}(\omega) 4 C^{\prime \prime 3}\left(\frac{t_{2}}{2}\right) C^{\prime \prime}\left(t_{1}+\frac{t_{2}}{2}\right)\right\},
\end{aligned}
$$

where the intensities of the nonlinear polarizability and anharmonicity are now denoted by $k_{a b c d e f, \mathrm{NL}}(\omega)$ and $k_{\text {abcdef,AN }}(\omega)$, respectively. As is illustrated below, $k_{a b c d e f, \mathrm{NL}}(\omega)$ and $k_{a b c d e f, \mathrm{AN}}(\omega)$ can be evaluated quantitatively from the experimental and simulation data with the use of the double Fourier transformation of the response function defined by

$$
\begin{aligned}
\widetilde{R}_{a b c d e f}^{(5)}\left(\omega_{2}, \omega_{1}\right)= & \int_{0}^{\infty} d t_{1} \sin \left(\omega_{1} t_{1}\right) \\
& \times \int_{0}^{\infty} d t_{2} \sin \left(\omega_{2} t_{2}\right) R_{a b c d e f}^{(5)}\left(t_{2}, t_{1}\right) \\
= & \operatorname{Im}\left\{\int_{0}^{\infty} d t_{1} e^{i \omega_{1} t_{1}}\right. \\
& \left.\times \operatorname{Im}\left\{\int_{0}^{\infty} d t_{2} e^{i \omega_{2} t_{2}} R_{\text {abcdef }}^{(5)}\left(t_{2}, t_{1}\right)\right\}\right\} .
\end{aligned}
$$

The substitution of Eq. (11) into Eq. (12) leads to the expression without tensor elements as

$$
\begin{aligned}
\widetilde{R}^{(5)}\left(\omega_{2}, \omega_{1}\right) \propto & \int d \omega \frac{\rho(\omega)}{\omega^{2}}\left\{k _ { \mathrm { NL } } ( \omega ) \left(\widetilde{R}_{0, \mathrm{NL}}\left(\omega_{2}, \omega_{1} ; \omega\right)\right.\right. \\
& \left.+\widetilde{R}_{1, \mathrm{NL}}\left(\omega_{2}, \omega_{1} ; \omega\right)+\widetilde{R}_{2, \mathrm{NL}}\left(\omega_{2}, \omega_{1} ; \omega\right)\right)+k_{\mathrm{AN}}(\omega) \\
& \times\left(\widetilde{R}_{0, \mathrm{AN}}\left(\omega_{2}, \omega_{1} ; \omega\right)+\widetilde{R}_{1, \mathrm{AN}}\left(\omega_{2}, \omega_{1} ; \omega\right)\right. \\
& \left.\left.+\widetilde{R}_{2, \mathrm{AN}}\left(\omega_{2}, \omega_{1} ; \omega\right)\right)\right\}
\end{aligned}
$$

where

$$
\begin{aligned}
& \widetilde{R}_{0, \mathrm{NL}}\left(\omega_{2}, \omega_{1} ; \omega\right)=\frac{1}{2} \frac{\left(\omega_{1}-\omega\right) \omega_{2}}{\left(\left(\omega_{1}-\omega\right)^{2}+\gamma^{2}\right)\left(\omega_{2}^{2}+4 \gamma^{2}\right)}, \\
& \widetilde{R}_{1, \mathrm{NL}}\left(\omega_{2}, \omega_{1} ; \omega\right)=\frac{\gamma^{2}}{\left(\left(\omega_{1}-\omega\right)^{2}+\gamma^{2}\right)\left(\left(\omega_{2}-\omega\right)^{2}+\gamma^{2}\right)}, \\
& \widetilde{R}_{2, \mathrm{NL}}\left(\omega_{2}, \omega_{1} ; \omega\right)=\frac{1}{2} \frac{2 \gamma^{2}-\left(\omega_{1}-\omega\right)\left(\omega_{2}-2 \omega\right)}{\left(\left(\omega_{1}-\omega\right)^{2}+\gamma^{2}\right)\left(\left(\omega_{2}-2 \omega\right)^{2}+4 \gamma^{2}\right)},
\end{aligned}
$$

$\tilde{R}_{0, \mathrm{AN}}\left(\omega_{2}, \omega_{1} ; \omega\right)=\frac{3}{2} \frac{\left(\omega_{1}-\omega\right) \omega_{2}}{\left(\left(\omega_{1}-\omega\right)^{2}+\gamma^{2}\right)\left(\omega_{2}^{2}+4 \gamma^{2}\right)}$,

$\widetilde{R}_{1, \mathrm{AN}}\left(\omega_{2}, \omega_{1} ; \omega\right)=\frac{\gamma^{2}-2\left(\omega_{1}-\omega\right)\left(\omega_{2}-\omega\right)}{\left(\left(\omega_{1}-\omega\right)^{2}+\gamma^{2}\right)\left(\left(\omega_{2}-\omega\right)^{2}+\gamma^{2}\right)}$,

and

$$
\widetilde{R}_{2, \mathrm{AN}}\left(\omega_{2}, \omega_{1} ; \omega\right)=\frac{1}{2} \frac{-2 \gamma^{2}+\left(\omega_{1}-\omega\right)\left(\omega_{2}-2 \omega\right)}{\left(\left(\omega_{1}-\omega\right)^{2}+\gamma^{2}\right)\left(\left(\omega_{2}-2 \omega\right)^{2}+4 \gamma^{2}\right)}
$$

If we set $\rho(\omega)=\delta\left(\omega-\omega_{a}\right)$, the signal consists of two peaks centered at $\omega_{1}=\omega_{2}=\omega_{a}$ and $\omega_{1}=\omega_{2} / 2=\omega_{a}$ as

$$
\begin{aligned}
\widetilde{R}_{\mathrm{obs}}^{(5)}\left(\omega_{2}, \omega_{1}\right) \propto & k_{\mathrm{NL}}\left(\omega_{a}\right) \frac{1}{\left(\omega_{1}-\omega_{a}\right)^{2}+\gamma^{2}} \\
& \times\left(\frac{1}{\left(\omega_{2}-\omega_{a}\right)^{2}+\gamma^{2}}+\frac{1}{2} \frac{2}{\left(\omega_{2}-2 \omega_{a}\right)^{2}+4 \gamma^{2}}\right) \\
& +k_{\mathrm{AN}}\left(\omega_{a}\right) \frac{1}{\left(\omega_{1}-\omega_{a}\right)^{2}+\gamma^{2}} \\
& \times\left(\frac{1}{\left(\omega_{2}-\omega_{a}\right)^{2}+\gamma^{2}}-\frac{1}{2} \frac{2}{\left(\omega_{2}-2 \omega_{a}\right)^{2}+4 \gamma^{2}}\right)
\end{aligned}
$$

In a real system the spectral distribution $\rho(\omega)$ may not be a delta function, and the terms such as

$$
\int d \omega \frac{\rho(\omega)}{\omega^{2}} \frac{\left(\omega_{1}-\omega\right)\left(\omega_{2}-\omega\right)}{\left(\left(\omega_{1}-\omega\right)^{2}+\gamma^{2}\right)\left(\left(\omega_{2}-\omega\right)^{2}+\gamma^{2}\right)}
$$

can also make a contributions to the spectra. Therefore the profiles and positions of peaks are slightly displaced from those predicted by Eq. (20). In the case that $\rho(\omega)$ has a Gaussian-type profile, however, the contributions of Eq. (21) to the spectral volume centered at $\omega \approx \omega_{a}$ is negligible because we have

$$
\begin{aligned}
& \int_{\omega_{a}-\Delta \omega}^{\omega_{a}+\Delta \omega} d \omega_{2} \int_{\omega_{a}-\Delta \omega}^{\omega_{a}+\Delta \omega} d \omega_{1} \int d \omega \frac{\rho(\omega)}{\omega^{2}} \\
& \times \frac{\left(\omega_{1}-\omega\right)\left(\omega_{2}-\omega\right)}{\left(\left(\omega_{1}-\omega\right)^{2}+\gamma^{2}\right)\left(\left(\omega_{2}-\omega\right)^{2}+\gamma^{2}\right)} \approx 0,
\end{aligned}
$$

where $\Delta \omega$ represents the spectral width. If a frequencyresolved spectrum obtained from either the experiments or simulations is expressed as

$$
\begin{aligned}
\widetilde{R}_{\mathrm{obs}}^{(5)}\left(\omega_{2}, \omega_{1}\right)= & \widetilde{R}_{\mathrm{obs}}^{(5)}\left(\omega_{2} \approx \omega_{a}, \omega_{1} \approx \omega_{a}\right) \\
& +\widetilde{R}_{\mathrm{obs}}^{(5)}\left(\omega_{2} \approx 2 \omega_{a}, \omega_{1} \approx \omega_{a}\right),
\end{aligned}
$$

the contributions from the nonlinear polarizability and anharmonicity for each molecular motion can be evaluated through the following equation:

$$
\frac{k_{\mathrm{AN}}\left(\omega_{a}\right)}{k_{\mathrm{NL}}\left(\omega_{a}\right)} \approx \frac{\widetilde{R}_{\mathrm{obs}}^{(5)}\left(\omega_{a}, \omega_{a}\right)-2 \widetilde{R}_{\mathrm{obs}}^{(5)}\left(2 \omega_{a}, \omega_{a}\right)}{\widetilde{R}_{\mathrm{obs}}^{(5)}\left(\omega_{a}, \omega_{a}\right)+2 \widetilde{R}_{\mathrm{obs}}^{(5)}\left(2 \omega_{a}, \omega_{a}\right)}
$$

We now consider the method to separate the peak near $\omega_{1}=\omega_{2}=\omega_{a}$ from other peaks, for example, near $\omega_{1}=\omega_{2} / 2$ $=\omega_{a}$. If $\widetilde{R}_{\mathrm{obs}}^{(5)}\left(\omega_{2}, \omega_{1}\right)-\widetilde{R}_{\mathrm{obs}}^{(5)}\left(\omega_{1}, \omega_{2}\right)$ is calculated, Eqs. (15) and (18) vanish because of the symmetry with respect to $\omega_{1}$ and $\omega_{2}$ for any $\rho(\omega)$. Moreover, by using $\widetilde{R}_{\mathrm{obs}}^{(5)}\left(\omega_{2}, \omega_{1}\right)$ $-\widetilde{R}_{\mathrm{obs}}^{(5)}\left(\omega_{1}, \omega_{2}\right)$, we can remove the cross peaks which often 

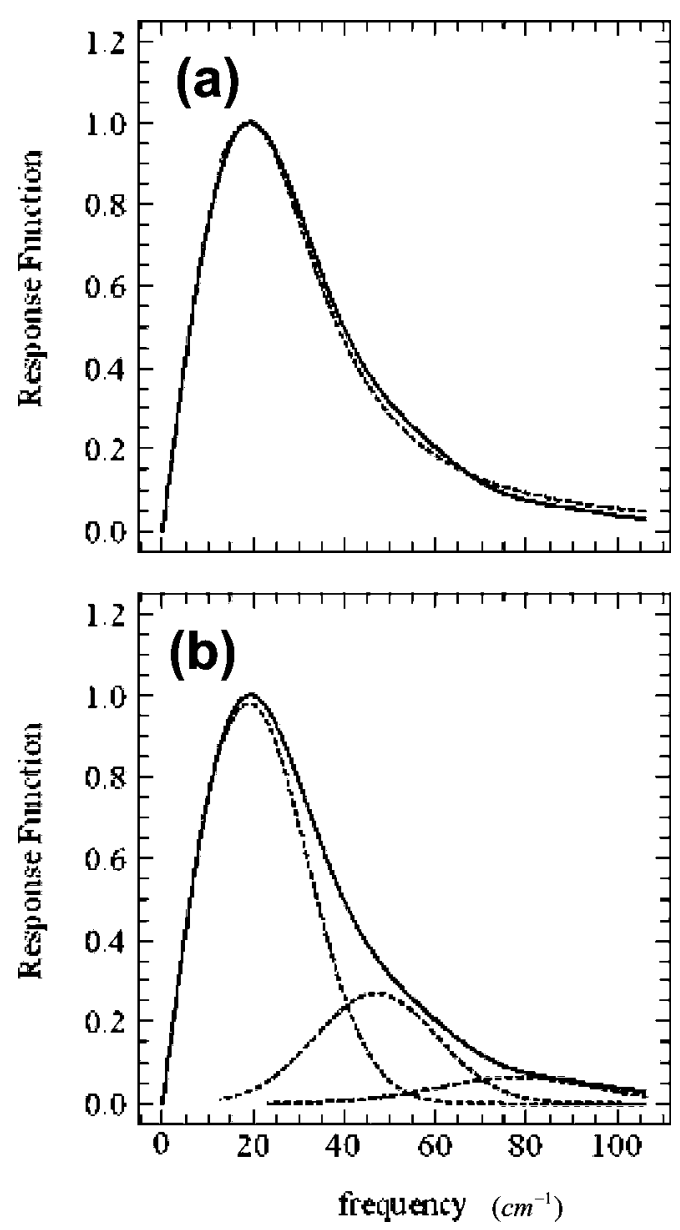

FIG. 1. The third-order response functions $\widetilde{R}^{(3)}(\omega)$ are depicted as the solid line. (a) The curve fitted with one Lorentzian peak and (b) the curves fitted with three Gaussian peaks are plotted as the dashed lines.

overlap with the peak at $\omega_{1}=\omega_{2} / 2$ in a multimode system. The volume of each peak can be measured accurately as illustrated in Secs. III and IV. Hereafter, we discuss abcd $=z z z z$ and $a b c d e f=z z z z z z$ tensors in the response functions and drop the suffixes for simplicity.

\section{ANALYZING THE TWO-DIMENSIONAL SIGNALS OF LENNARD-JONES SYSTEMS}

Using 2D frequency domain maps, we can also clarify the peaks more clearly than 1D frequency domain plots. In this section, we apply our method to the fifth-order 2D Raman signals of the LJ potential system, which were well investigated by Ma and Stratt, ${ }^{14,15}$ to evaluate the consistency of the ratio $k_{\mathrm{AN}} / k_{\mathrm{NL}}$. At the same time, we demonstrate a way to judge whether the spectral peak is composed of a single mode or multimodes.

We calculate the fifth-order Raman signals in the LJ potential system by the equilibrium and nonequilibrium hybrid methods. ${ }^{13}$ Then, we transformed the signals from time domain to frequency domain with a Welch window function. MD simulations are carried out with $108 \mathrm{LJ}$ atoms. The same potential, polarizability, size of the simulation box, and time step used in these simulations are used as those of Ma and Stratt's simulation. ${ }^{14,15}$ The forces by LJ potential and DID interaction are smoothly cut off at the half length of the simulation box with the switching function. In the nonequilibrium calculations, the system is irradiated by laser pulse pairs with the strength of $5.0 \mathrm{~V} / \AA$. The third-and fifth-order response functions are calculated by averaging over 4000000 configurations.

The 1D frequency domain plot of the third-order response function $\widetilde{R}^{(3)}(\omega)$ is shown in Fig. 1. We found that the signal may be fitted by either one Lorentzian peak or three Gaussian peaks, which are depicted in Figs. 1(a) and 1(b), respectively. ${ }^{26}$ Figures $2(\mathrm{a})$ and $2(\mathrm{~b})$ show the $2 \mathrm{D}$ contour plots in frequency domain, $\widetilde{R}^{(5)}\left(\omega_{2}, \omega_{1}\right)$ and $\widetilde{R}^{(5)}\left(\omega_{2}, \omega_{1}\right)$ $-\widetilde{R}^{(5)}\left(\omega_{1}, \omega_{2}\right)$, respectively. These figures indicate that the dynamics in the LJ potential system is governed by one mode. Thus, $\hat{R}^{(3)}(\omega)$ should be fitted with a single Gaussian peak as Fig. 1(b), and the frequency for the translational motion is found to be about $19 \mathrm{~cm}^{-1}$.

As shown in Fig. 2(b), $\widetilde{R}^{(5)}\left(\omega_{2}, \omega_{1}\right)-\widetilde{R}^{(5)}\left(\omega_{1}, \omega_{2}\right)$ allows us to specify the peak near $\omega_{1}=\omega_{2} / 2=19 \mathrm{~cm}^{-1}$ more easily than $\widetilde{R}^{(5)}\left(\omega_{2}, \omega_{1}\right)$ does. The calculated ratio of the spectral volumes, $\widetilde{R}^{(5)}\left(\omega_{1}=\omega_{2} / 2\right) / \widetilde{R}^{(5)}\left(\omega_{1}=\omega_{2}\right)$, is -0.30 , which gives us $k_{\mathrm{AN}} / k_{\mathrm{NL}}=4.0$ for the translational mode. In fact, if we adapt this ratio and calculate the diagonal element on $t_{1}=t_{2}$ and $t_{2}$ axis element on $t_{1}=0$ of Eq. (10) according to the NM expressions using the third-order response function $R^{(3)}(t)$ as $^{11}$

$$
\begin{aligned}
R^{(5)}(t, t) \propto & k_{\mathrm{NL}} \int_{0}^{t} d t\left(\frac{3}{2} R^{(3)}(3 t)+R^{(3)}(2 t)-\frac{1}{2} R^{(3)}(t)\right) \\
& +k_{\mathrm{AN}} \int_{0}^{t} d t\left(-3 R^{(3)}(3 t)+6 R^{(3)}(2 t)-3 R^{(3)}(t)\right)
\end{aligned}
$$

and

$R^{(3)}(t, 0) \propto k_{\mathrm{NL}} \int_{0}^{t} d t R^{(3)}(2 t)+k_{\mathrm{AN}} \int_{0}^{t} d t\left(-R^{(3)}(2 t)+2 R^{(3)}(t)\right)$,

we can well reconstruct the original fifth-order response function in the short time region as plotted in Fig. 2(c). The large deviations of Eqs. (25) and (26) from MD simulations over $200 \mathrm{fs}$ are attributed to the use of the NM expression instead of BO expression. Moreover, the agreement of these results with Fig. 7 in Ref. 15 strongly supports the ability to evaluate the ratio between anharmonicity and nonlinearity from our present method.

The temperature dependence of the ratio $k_{\mathrm{NL}} / k_{\mathrm{AN}}$ is investigated at $T=95,120$, and $160 \mathrm{~K}$ for the supercooled liquids and at $T=220$ and $260 \mathrm{~K}$ for the normal liquids under same density and is shown in Fig. 3. ${ }^{35}$ In Fig. 3, this ratio seems almost constant in both phases, which means that fifth-order response function cannot capture the qualitative change between both liquids. 

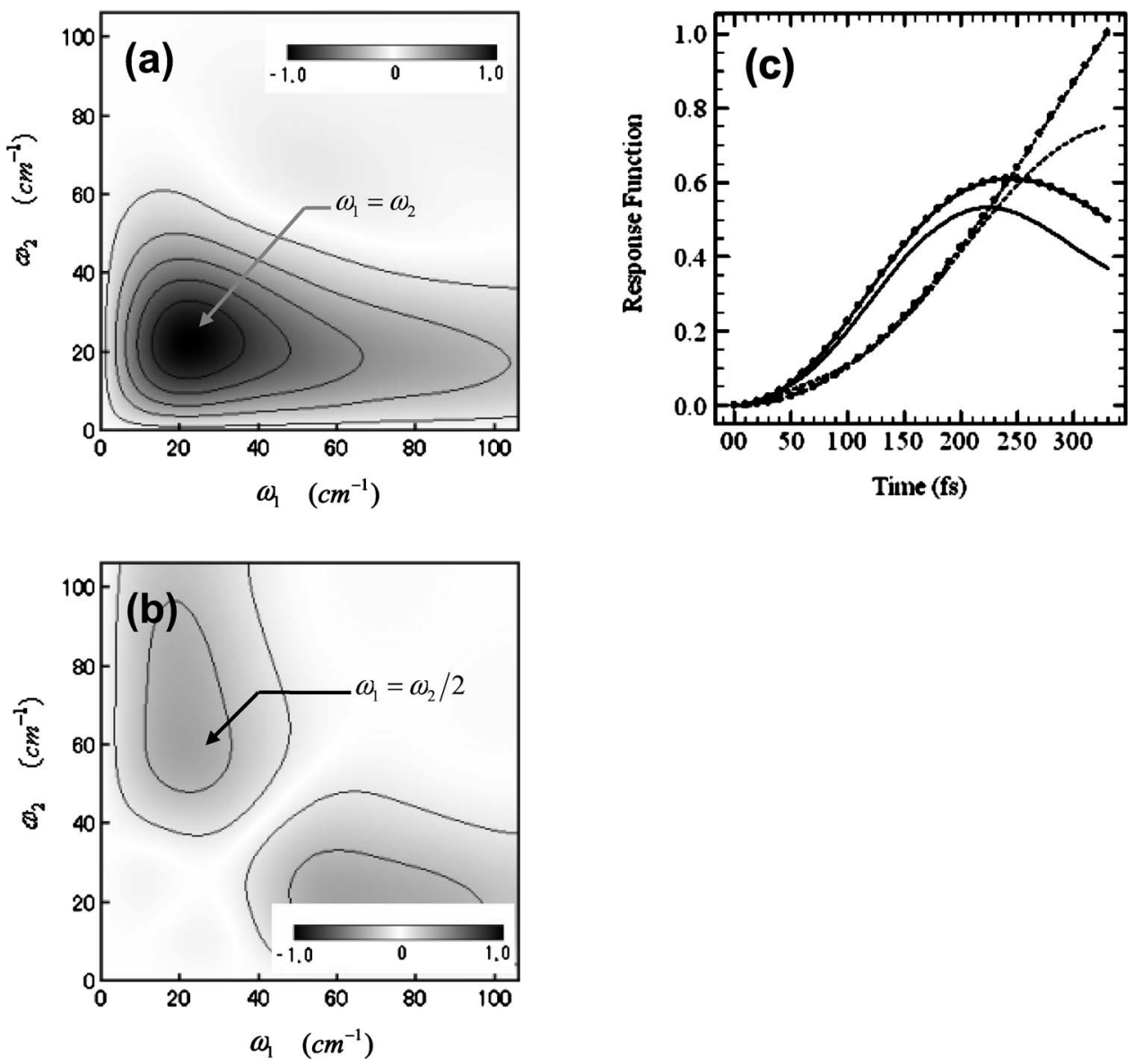

FIG. 2. (a) The fifth-order response function $\widetilde{R}^{(5)}\left(\omega_{2}, \omega_{1}\right)$ and (b) $\widetilde{R}^{(5)}\left(\omega_{2}, \omega_{1}\right)-\widetilde{R}^{(5)}\left(\omega_{1}, \omega_{2}\right)$ for the LJ system at $T=220 \mathrm{~K}$ are depicted. In Fig. (c), the MD results along $t_{1}=t_{2}$ (solid line) and $t_{1}=0$ (dotted line) are compared with the signals calculated from Eqs. (25) (solid line with circle markers) and (26) (dotted line with circle markers), respectively.

\section{ANALYZING THE TWO-DIMENSIONAL SIGNALS OF SOFT-CORE POTENTIAL SYSTEMS}

We investigate the soft-core potential system defined by the potential

$$
U(r)=\varepsilon\left(\frac{\sigma}{r}\right)^{6}+A\left(\frac{r}{\sigma}\right)^{4}+B
$$

where $\varepsilon$ and $\sigma$ are the potential parameters and the constants $A$ and $B$ are chosen to connect the force and potential smoothly at the cutoff $r_{0}$. Thus $A$ and $B$ are given by $(3 / 2) \varepsilon\left(\sigma / r_{0}\right)^{10}$ and $(-5 / 2) \varepsilon\left(\sigma / r_{0}\right)^{6}$, respectively. We carried out the MD simulation in reduced units. A temperature multiplied by the Boltzmann constant is changed from solid phase $(k T=0.14,0.155)$ to liquid phase $(k T$ $=0.20,0.215,0.23)$ as a parameter with fixed density. The other conditions on the simulation are the same as in the previous study, ${ }^{11}$ in which we found that the fifth-order Raman signals are sensitive to the difference of the phases while the third-order Raman signals are not. The method developed in this paper sheds light on the relative intensities of the nonlinear polarizability and anharmonicity on the fifth-order signals.

The 1D time domain and frequency domain plots, $R^{(3)}(t)$ and $\widetilde{R}^{(3)}(\omega)$, 2D time domain and frequency domain maps, $\quad R^{(5)}\left(t_{2}, t_{1}\right) \quad$ and $\quad \widetilde{R}^{(5)}\left(\omega_{2}, \omega_{1}\right), \quad$ and $\quad \widetilde{R}^{(5)}\left(\omega_{2}, \omega_{1}\right)$
$-\widetilde{R}^{(5)}\left(\omega_{1}, \omega_{2}\right)$ at $k T=0.14$ and 0.20 are depicted Figs. 4(a)-4(e) and Figs. 5(a)-5(e), respectively. Figure 4(b) exhibits a similar profile to Fig. 5(b) and the 1D frequency domain maps in the other temperatures. These signals are also similar to the LJ case depicted in Fig. 1. On the other hand, we can clearly see the difference of $2 \mathrm{D}$ signals in the soft-core and LJ cases; the 2D signals of soft-core potential system consist of two modes. The frequencies of two modes

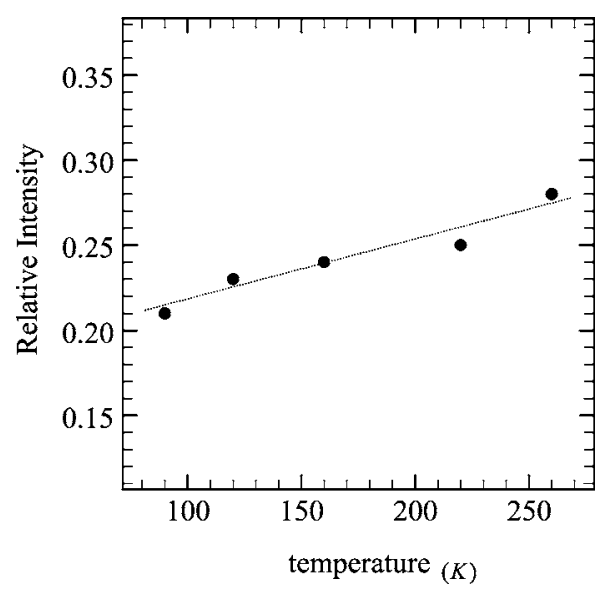

FIG. 3. The ratio of $k_{\mathrm{NL}} / k_{\mathrm{AN}}$ for the LJ potential system is illustrated as the function of temperature. The dashed line is a guide for eyes. 

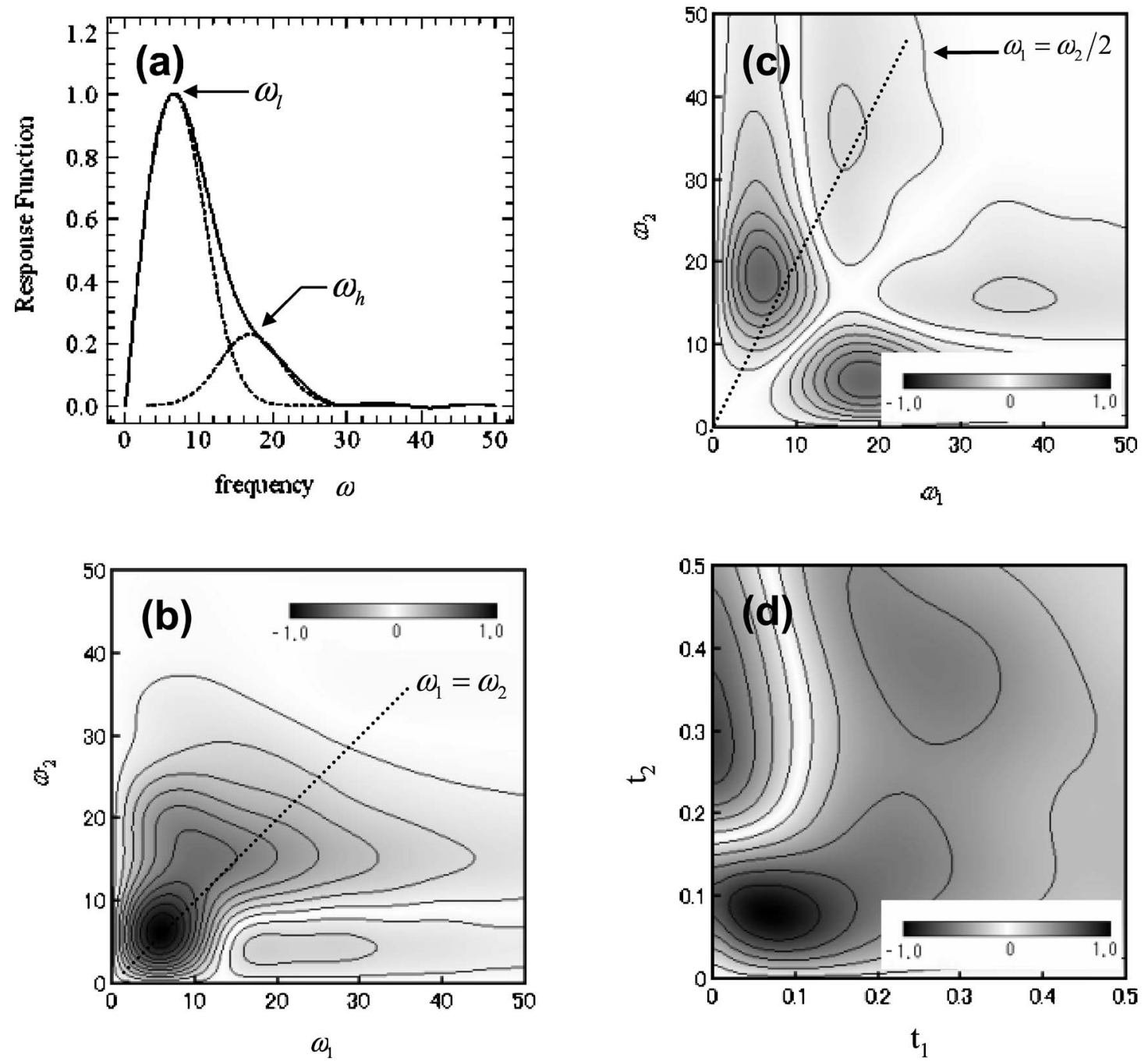

FIG. 4. (a) The third-order response function $\widetilde{R}^{(3)}(\omega)$ and the fifth-order response functions (b) $\widetilde{R}^{(5)}\left(\omega_{2}, \omega_{1}\right)$ and (c) $\widetilde{R}^{(5)}\left(\omega_{2}, \omega_{1}\right)-\widetilde{R}^{(5)}\left(\omega_{1}, \omega_{2}\right)$ for the soft-core system at $k T=0.14$ are depicted. (d) The time domain $2 \mathrm{D}$ maps $R^{(5)}\left(t_{2}, t_{1}\right)$ are shown for comparison (see Ref. 11). The curve fitted to the third-order spectrum with two Gaussian peaks is presented as the dotted line. All figures are in reduced units.

are found to be $\omega_{l}=6.5$ and $\omega_{h}=16$, respectively. The modes of $\omega_{l}$ and $\omega_{h}$ are thought to be from delocalized vibrational motion for each atom and high frequency localized mode, respectively. ${ }^{36}$

The relative intensities of the nonlinear polarizability and anharmonicity for $\omega_{l}$ and $\omega_{h}$ are determined from the spectral volumes of $2 \mathrm{D}$ frequency domain maps as summarized in Table I. The calculated ratios for each mode are visualized in Fig. 6 for $k_{\mathrm{NL}}\left(\omega_{l}\right) / k_{\mathrm{AN}}\left(\omega_{l}\right), k_{\mathrm{AN}}\left(\omega_{h}\right) / k_{\mathrm{AN}}\left(\omega_{l}\right)$, and $k_{\mathrm{AN}}\left(\omega_{h}\right) / k_{\mathrm{AN}}\left(\omega_{h}\right)$. All ratios except for the nonlinear polarizability at $\omega_{l}$ show almost proportional to each other at any temperature, while the ratios between $k_{\mathrm{NL}}\left(\omega_{l}\right)$ and the other intensities exhibit a specific phase dependency as shown in Fig. 6. Thus, we may consider that the nonlinear polarizability of the delocalized mode is dramatically changed, whereas the high frequency localized mode is not changed as long as the fifth-order Raman spectra can capture. This consideration indicates that the difference of the antisymmetric integrated response functions between solid and liquid phases ${ }^{11}$ is induced by the nonlinear polarizability of $\omega_{l}$. In addition, the anharmonicity makes larger contribution to the fifth-order signal than the nonlinear polarizability for the localized mode while making smaller contribution for delocalized mode as shown in Table I.

\section{CONCLUSION}

In this paper, we proposed the method to evaluate the contributions from the nonlinear polarizability and anharmonicity of potentials utilizing the analytical expression of 2D signals in frequency domain. The ratio between two contributions was evaluated from the volumes of spectral peaks. With 2D Raman frequency domain maps, we could easily separate contributions from different vibrational modes, which was difficult with 1D approach.

Demonstration to apply our method to the simulation results for LJ potential system indicates that primary contribution of molecular motions to the signal comes from only a translational mode at $19 \mathrm{~cm}^{-1}$. Moreover we evaluated the ratio between nonlinear polarizability and anharmonicity as $k_{\mathrm{AN}} / k_{\mathrm{NL}}=4.0$. Good agreement is obtained in comparison of 

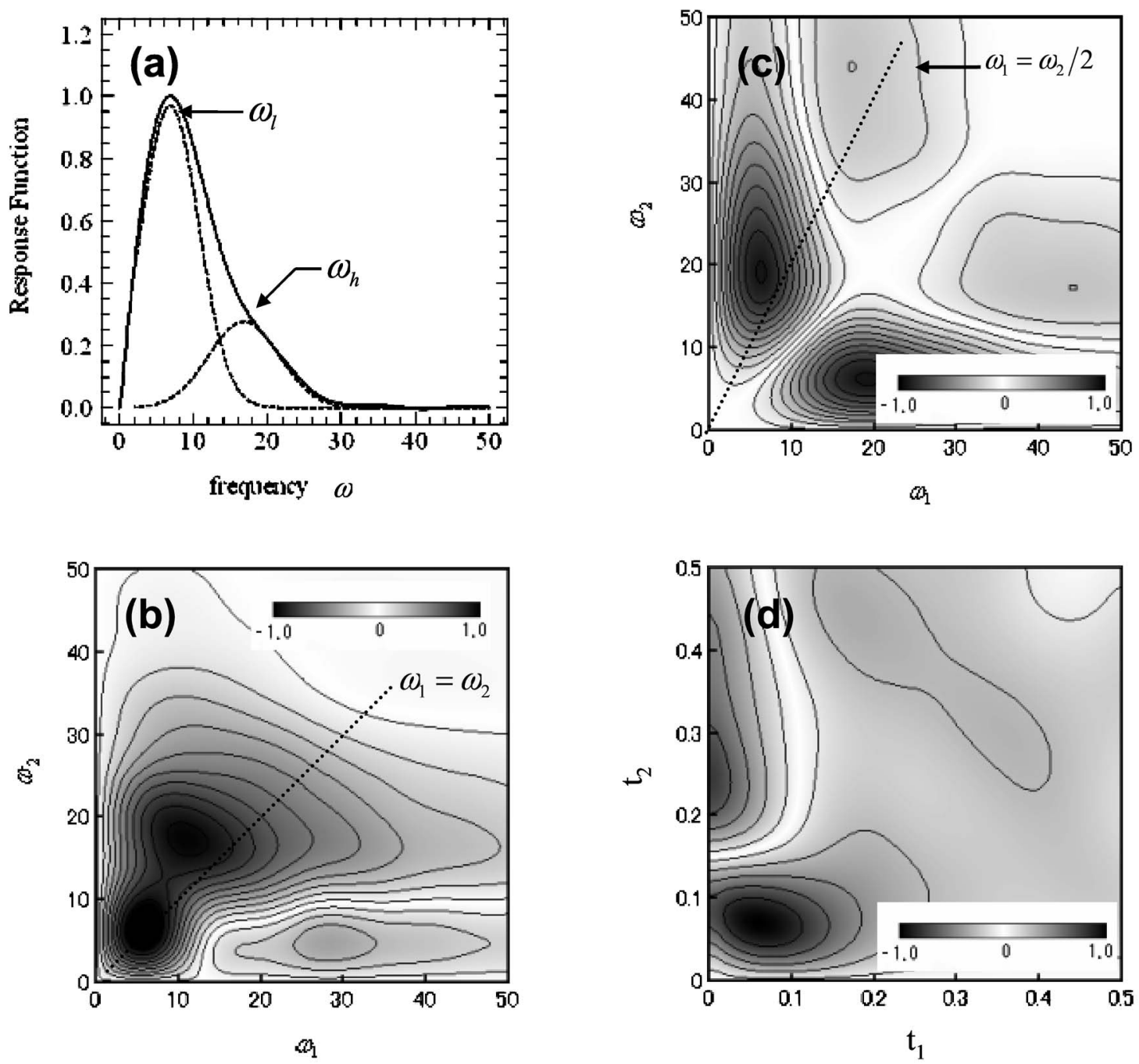

FIG. 5. (a) The third-order response function $\widetilde{R}^{(3)}(\omega)$ and the fifth-order response functions (b) $\widetilde{R}^{(5)}\left(\omega_{2}, \omega_{1}\right)$ and (c) $\widetilde{R}^{(5)}\left(\omega_{2}, \omega_{1}\right)-\widetilde{R}^{(5)}\left(\omega_{1}, \omega_{2}\right)$ for the soft-core system at $k T=0.20$ are depicted. (d) The time domain $2 \mathrm{D}$ maps $R^{(5)}\left(t_{2}, t_{1}\right)$ are shown for comparison (see Ref. 11). The curve fitted to the third-order spectrum with two Gaussian peaks is presented as the dotted line. All figures are in reduced units.

the NM expression of the fifth-order response functions using this ratio with the data of Ma and Stratt, ${ }^{15}$ which supports the consistency of our method.

We also examined our method to analyze the signals of the soft-core potential system at various temperatures. In our previous study, ${ }^{11}$ it was found that the $2 \mathrm{D}$ time domain Raman signals exhibited a clear difference between the solid and liquid phases, but the origin of this difference could not be identified. The present method shows that the different

TABLE I. Relative ratios between anharmonicity of potentials and nonlinear of polarizability.

\begin{tabular}{cccccc}
\hline \hline & \multicolumn{3}{c}{$\omega_{l}$} & & \multicolumn{2}{c}{$\omega_{h}$} \\
\cline { 2 - 3 } \cline { 5 - 6 } $\begin{array}{c}\text { Temperature } \\
(k T)\end{array}$ & $\mathrm{AN}$ & $\mathrm{NL}$ & & $\mathrm{AN}$ & $\mathrm{NL}$ \\
\hline 0.140 & 2.6 & -0.24 & & 0.25 & 1.0 \\
0.155 & 2.5 & -0.21 & & 0.25 & 1.0 \\
0.200 & 2.0 & -0.46 & & 0.34 & 1.0 \\
0.215 & 2.2 & -0.82 & & 0.35 & 1.0 \\
0.230 & 2.1 & -0.97 & & 0.39 & 1.0 \\
\hline \hline
\end{tabular}

fifth-order Raman signals between both phases resulted from the change of the mode of $\omega_{l}$ through the nonlinear polarizability. At the same time, the phase transition was shown to have little effect on the mode of $\omega_{h}$. We can conclude from

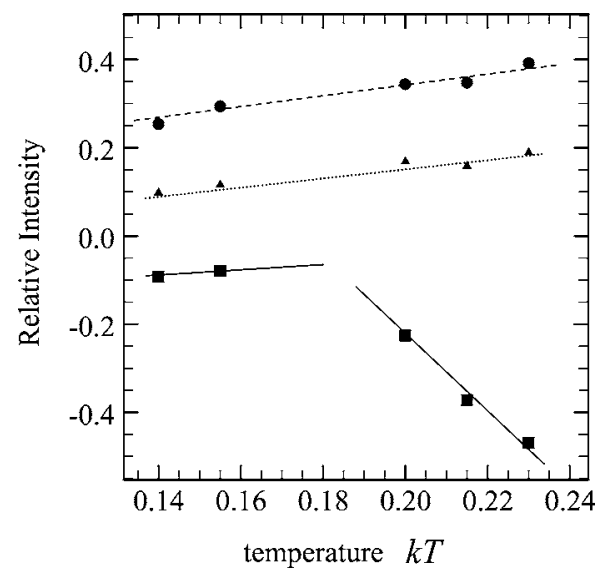

FIG. 6. The ratios of $k_{\mathrm{NL}}\left(\omega_{l}\right) / k_{\mathrm{AN}}\left(\omega_{l}\right)$ (square), $k_{\mathrm{AN}}\left(\omega_{h}\right) / k_{\mathrm{AN}}\left(\omega_{l}\right)$ (triangle), and $k_{\mathrm{AN}}\left(\omega_{h}\right) / k_{\mathrm{NL}}\left(\omega_{h}\right)$ (circle) for the soft-core potential system are illustrated as the function of temperature. All lines are guides for eyes. 
the fifth-order response function that the dynamical change between the solid and liquid phases is characterized not as high frequency localized mode but as delocalized motion in soft-core potential systems. Moreover when our attention is paid to the contributions to the fifth-order signal, we can find that the nonlinear polarizability and anharmonicity of potential are dominant for the localized and delocalized modes, respectively.

\section{ACKNOWLEDGMENTS}

We wish to express our gratitude to Prof. Dwayne Miller and Mr. Chris Milne for fruitful discussion. One of the authors (Y.T.) is thankful for the financial support from a Grant-in-Aid for Scientific Research A 15205005 from Japan Society for the Promotion of Science and Morino Science Foundation. Some parts of the numerical calculations were performed at the Computer Center of the Institute for Molecular Science.

\section{APPENDIX}

The contribution of anharmonicity to the fifth-order Raman signal was first evaluated by Okumura and Tanimura using Feynman rules for anharmonicity additive potential. ${ }^{3}$ Then, by using the adiabatic instantaneous normal mode theory, Ma and Stratt $^{15}$ derived the same expression as Okumura and Tanimura. The validity of the expression was well confirmed for a case of Morse potential system from FokkerPlanck equation approach. ${ }^{6}$ Here, we show that the same expression can be obtained from the perturbative calculations by assuming a Morse potential system defined by Eq. (8). When the correspondence of the anharmonicity additive potential

$$
V(q)=\frac{\mu \omega^{2}}{2} q^{2}(t)+\frac{g_{3}}{3 !} q^{3}(t)
$$

with the Morse potential is taken into consideration, we can obtain the relations $\omega=\beta \sqrt{2 D / \mu}$ and $g_{3}=-6 D \beta^{3}$ by expanding the Morse potential with respect to $q$. We may evaluate the fifth-order response function from the solution of the Morse potential, because the contributions of the nonlinear polarizability and anharmonicity over $\lambda^{2}$ are negligible at small $q$.

The equation of motion for the Morse potential system can be solved analytically and the coordinate and momentum are given by

$$
q(t)=\frac{1}{\beta} \ln \frac{1+\sqrt{1-C_{1}} \sin \left(\sqrt{2 D C_{1} / \mu} \beta t+C_{2}\right)}{C_{1}}
$$

and

$$
p(t)=\sqrt{2 \mu D C_{1}} \frac{\sqrt{1-C_{1}} \cos \left(\sqrt{2 D C_{1} / \mu} \beta t+C_{2}\right)}{1+\sqrt{1-C_{1}} \sin \left(\sqrt{2 D C_{1} / \mu} \beta t+C_{2}\right)},
$$

where $\mu$ is the mass of molecule and $C_{1}$ and $C_{2}$ are the integral constants. When we expand Taylor series up to the first-order around the bottom of the Morse potential, the position and momentum are written as

$$
q(t)=\frac{1}{\beta}\left(\sqrt{1-C_{1}} \sin \left(\sqrt{\frac{2 D}{\mu}} \beta t+C_{2}\right)-\frac{1-C_{1}}{2}\left(-2+\sin ^{2}\left(\sqrt{\frac{2 D}{\mu}} \beta t+C_{2}\right)\right)+\cdots\right)
$$

and

$$
p(t)=\sqrt{2 D \mu}\left(\sqrt{1-C_{1}} \cos \left(\sqrt{\frac{2 D}{\mu}} \beta t+C_{2}\right)-\frac{1-C_{1}}{2} \sin \left(2\left(\sqrt{\frac{2 D}{\mu}} \beta t+C_{2}\right)\right)+\cdots\right) .
$$

Using these solutions, we can calculate the stability matrix analytically as

$$
\frac{\partial q(t)}{\partial p(0)}=\frac{1}{\beta \sqrt{2 D \mu}}\left(\sin \left(\sqrt{\frac{2 D}{\mu}} \beta t\right)+4 \sqrt{1-C_{1}} \sin \left(\sqrt{\frac{D}{2 \mu}} \beta t+C_{2}\right) \sin ^{3}\left(\sqrt{\frac{D}{2 \mu}} \beta t\right)+\cdots\right) .
$$

Thus

$$
\begin{aligned}
& q_{H}(t)=\frac{\sqrt{1-C_{1}}}{\beta} \sin \left(\sqrt{\frac{2 D}{\mu}} \beta t+C_{2}\right), \\
& q_{A}(t)=-\frac{1-C_{1}}{2 \beta}\left(-2+\sin ^{2}\left(\sqrt{\frac{2 D}{\mu}} \beta t+C_{2}\right)\right), \\
& M_{H}(t)=\frac{1}{\beta \sqrt{2 D \mu}} \sin \left(\sqrt{\frac{2 D}{\mu}} \beta t\right),
\end{aligned}
$$

and 


$$
M_{A}(t)=\frac{4 \sqrt{1-C_{1}}}{\beta \sqrt{2 D \mu}} \sin \left(\sqrt{\frac{D}{2 \mu}} \beta t+C_{2}\right) \sin ^{2}\left(\sqrt{\frac{D}{2 \mu}} \beta t\right) .
$$

The substitution of these equations into Eqs. (3)-(6) gives us the expression for the anharmonicity of potentials as

$$
\begin{aligned}
R_{a b c d e f}^{(5)}\left(t_{2}, t_{1}\right)= & \frac{1}{k T}\left\langle\Pi_{a b}^{\prime \prime} \Pi_{c d}^{\prime} \Pi_{e f}^{\prime} \frac{\left(1-C_{1}\right)}{\mu \beta^{2}} \sin \left(\sqrt{\frac{2 D}{\mu}} \beta t_{2}\right) \sin \left(\sqrt{\frac{2 D}{\mu}} \beta\left(t_{1}+t_{2}\right)\right)\right\rangle \\
& +\frac{1}{k T}\left\langle\Pi_{a b}^{\prime} \Pi_{c d}^{\prime \prime} \Pi_{e f}^{\prime} \frac{\left(1-C_{1}\right)}{\mu \beta^{2}} \sin \left(\sqrt{\frac{2 D}{\mu}} \beta t_{2}\right) \sin \left(\sqrt{\frac{2 D}{\mu}} \beta t_{1}\right)\right\rangle \\
& +\frac{1}{k T}\left\langle\Pi_{a b}^{\prime} \Pi_{c d}^{\prime} \Pi_{e f}^{\prime} \frac{4\left(1-C_{1}\right)}{\mu \beta} \sin ^{3}\left(\sqrt{\frac{2 D}{\mu}} \beta t_{2}\right) \sin \left(\sqrt{\frac{2 D}{\mu}} \beta t_{1}+\sqrt{\frac{D}{2 \mu}} \beta t_{2}\right)\right\rangle .
\end{aligned}
$$

When the relations $\omega=\beta \sqrt{2 D / \mu}$ and $\left(1-C_{1}\right) / D=\mu \omega^{2} q^{2} / 2=k T / 2$ are used, Eq. (A11) becomes

$$
\begin{aligned}
R_{a b c d e f}^{(5)}\left(t_{2}, t_{1}\right) \propto & \left\langle\Pi_{a b}^{\prime \prime} \Pi_{c d}^{\prime} \Pi_{e f}^{\prime} \frac{1}{\omega^{2}} \sin \left(\omega t_{2}\right) \sin \left(\omega t_{1}+\omega t_{2}\right)\right\rangle+\left\langle\Pi_{a b}^{\prime} \Pi_{c d}^{\prime \prime} \Pi_{e f}^{\prime} \frac{1}{\omega^{2}} \sin \left(\omega t_{2}\right) \sin \left(\omega t_{1}\right)\right\rangle \\
& -\left\langle\Pi_{a b}^{\prime} \Pi_{c d}^{\prime} \Pi_{e f}^{\prime} \frac{4 \beta}{\omega^{2}} \sin ^{3}\left(\omega t_{2}\right) \sin \left(\omega t_{1}+\frac{\omega}{2} t_{2}\right)\right\rangle .
\end{aligned}
$$

The expression for the anharmonicity additive potential is obtained by setting $g_{3}=-6 D \beta^{3}$, which has the same form as the expressions obtained by Okumura and Tanimura and by Ma and Stratt.

${ }^{1}$ S. Mukamel, Principles of Nonlinear Optical Spectroscopy (Oxford University Press, New York, 1995).

${ }^{2}$ Y. Tanimura and S. Mukamel, J. Chem. Phys. 99, 9496 (1993).

${ }^{3}$ K. Okumura and Y. Tanimura, J. Chem. Phys. 107, 2267 (1997).

${ }^{4}$ J. L. Wu and J. S. Cao, J. Chem. Phys. 115, 5381 (2001); M. Kryvohuz and J. S. Cao, Phys. Rev. Lett. 95, 180405 (2005).

${ }^{5}$ O. Kühn and Y. Tanimura, J. Chem. Phys. 119, 2155 (2003); T. Kato and Y. Tanimura, ibid. 120, 260 (2004); A. Ishizaki and Y. Tanimura, ibid. 123, 014503 (2005).

${ }^{6}$ Y. Tanimura, Chem. Phys. 233, 217 (1998); J. Phys. Soc. Jpn. (accepted).

${ }^{7}$ A. G. Dijkstra and J. Knoester, J. Phys. Chem. B 109, 9787 (2005).

${ }^{8}$ S. Saito and I. Ohmine, J. Chem. Phys. 108, 240 (1998).

${ }^{9}$ S. Saito and I. Ohmine, Phys. Rev. Lett. 88, 207401 (2002).

${ }^{10}$ S. Saito and I. Ohmine, J. Chem. Phys. 119, 9073 (2003).

${ }^{11}$ Y. Nagata and Y. Tanimura, J. Chem. Phys. 124, 024508 (2006).

${ }^{12}$ T. L. C. Jansen, J. G. Snijders, and K. Duppen, J. Chem. Phys. 113, 307 (2000); 114, 10910 (2001); T. I. C. Jansen, K. Duppen, and J. G. Snijders, Phys. Rev. B 67, 134206 (2003).

${ }^{13} \mathrm{~T}$. Hasegawa and Y. Tanimura (submitted).

${ }^{14}$ A. Ma and R. M. Stratt, Phys. Rev. Lett. 85, 1004 (2000).

${ }^{15}$ A. Ma and R. M. Stratt, J. Chem. Phys. 116, 4972 (2002).

${ }^{16}$ R. DeVane, C. Ridley, B. Space, and T. Keyes, J. Chem. Phys. 119, 6073 (2003).

${ }^{17}$ J. Kim and T. Keyes, Phys. Rev. E 65, 061102 (2002).T. Keyes and J. T. Fourkas, J. Chem. Phys. 112, 287 (2000).R. L. Murry, J. T. Fourkas, and T. Keyes, J. Chem. Phys. 109, 7913 (1998).

${ }^{18}$ M. Cho, in Advances in Multi-Photon Process and Spectroscopy, edited by S. H. Lin, A. A. Villaeys, and Y. Fujimura (World Scientific, Singapore, 1999), Vol. 12, p. 229.

${ }^{19}$ A. Piryatinski, C. P. Lawrence, and J. L. Skinner, J. Chem. Phys. 118, 9664 (2003); 118, 9672 (2003).
${ }^{20}$ R. Akiyama and R. F. Loring, J. Chem. Phys. 116, 4655 (2002); W. G. Noid and R. F. Loring, ibid. 121, 7057 (2004).

${ }^{21}$ M. F. Gelin D. Egorova, and W. Domcke, J. Chem. Phys. 123, 164112 (2005).

${ }^{22}$ A. M. Moran, S.-M. Park, and S. Mukamel, J. Chem. Phys. 118, 9971 (2003); T. Hayashi, T. L. C. Jansen, W. Zhuang, and S. Mukamel, J. Phys. Chem. A 109, 64 (2005).

${ }^{23}$ S. Hahn, K. Park, and M. Cho, J. Chem. Phys. 111, 4121 (1999).

${ }^{24}$ K. Tominaga and K. Yoshihara, Phys. Rev. Lett. 74, 3061 (1995); J. Chem. Phys. 104, 4419 (1996).

${ }^{25}$ V. Astinov, K. J. Kubarych, C. J. Milne, and R. J. D. Miller, Chem. Phys. Lett. 327, 334 (2000); K. J. Kubarych, C. L. Milne, S. Lin, V. Astinov, and R. J. D. Miller, J. Chem. Phys. 116, 2016 (2002); K. J. Kubarych, C. J. Milne, and R. J. D. Miller, Chem. Phys. Lett. 369, 635 (2003).

${ }^{26}$ A. Tokmakoff and G. R. Fleming, J. Chem. Phys. 106, 2569 (1997).

${ }^{27}$ D. A. Blank, L. J. Kaufman, and G. R. Fleming, J. Chem. Phys. 113, 771 (2000).

${ }^{28}$ L. J. Kaufman, D. A. Blank, and G. R. Fleming, J. Chem. Phys. 114, 2312 (2001); L. J. Kaufman, J. Heo, L. D. Ziegler, and G. R. Fleming, Phys. Rev. Lett. 88, 207402 (2002).

${ }^{29}$ D. Zimdars, A. Tokmakoff, S. Chen, S. R. Greenfield, M. D. Fayer, T. I. Smith, and H. A. Schwettman, Phys. Rev. Lett. 70, 2718 (1993).

${ }^{30}$ P. Hamm, M. Lin, and R. M. Hochstrasser, Phys. Rev. Lett. 81, 5326 (1998).

${ }^{31}$ E. C. Fulmer, F. Ding, P. Mukherjee, and M. T. Zanni, Phys. Rev. Lett. 94, 067402 (2005).

${ }^{32}$ K. J. Kubarych, C. J. Milne, and R. J. D. Miller, Int. Rev. Phys. Chem. 22, 497 (2003).

${ }^{33}$ G. R. Fleming, T. Joo, M. Cho, A. H. Zewail, V. S. Letokhov, R. A. Marcus, E. Pollak, D. J. Tannor, and S. Mukamel, Adv. Chem. Phys. 101, 141 (1997).

${ }^{34}$ J. Cao, S. Yang, and J. Wu, J. Chem. Phys. 116, 3760 (2002).

${ }^{35}$ The supercooled liquids at $T=95,120$, and $160 \mathrm{~K}$ appear as metastable states. The detailed phase diagrams are shown in J.-P. Hansen and L. Verlet, Phys. Rev. 184, 151 (1969); G. A. Vliegenthart, J. F. M. Lodge, and H. N. W. Lekkerkerker, Physica A 263, 378 (1999).

${ }^{36}$ H. R. Schober and B. B. Laird, Phys. Rev. B 44, 6746 (1991). 